\title{
Comic relief
}

$\mathrm{F}$ or much of 2008, Sarah lived alone in a small room on the schizophrenia ward at the Centre for Addiction and Mental Health in downtown Toronto. As she experienced psychosis, depression, and the solitude of her stark surroundings, Sarah found refuge in a resurrected passion for creating comics, and began posting a series online based on her experiences.

At that time, she couldn't have guessed that her work exemplified a growing trend in health care: fostering empathy and understanding through graphic art. During the recent University of Toronto conference, Comics \& Medicine: Navigating the Margins, Sarah (now known as Sarafin) presented a paper on her experiences and met artists, health care professionals, academics and patients who share a passion for graphic storytelling for various reasons: to make sense of illness, to bring meaning to their medical experience and to hone empathetic skills.

Graphic medicine is a substream of narrative medicine, which is used by health care professionals to "absorb, metabolize, interpret and be moved by stories of illness. ${ }^{1}$ Graphic narratives offer an accessible medium for patients and care providers alike. Those receiving care are often dealing with pain, fear and uncertainty; those providing it must maintain a sense of pathos and optimism despite witnessing suffering on a daily basis. A few simple drawings and words can capture, in the words of Dr. Michael Green, who teaches graphic medicine at Penn State University in Hershey, Pennsylvania, "profound experiences in an economical format."

Graphic narratives depicting the patient experience are commonly about the limitations of health care professionals and medical interven- illness" at the exclusion of "understanding the patient experience of illness. ... Frankly, I don't see these things as separate."

In a 2010 paper on graphic medicine, Green and Kimberly R. Myers argue that the process of reading a comic involves understanding "not only what is overtly seen and said but also what is implied" in the margins, or "gutters" between frames. "Thus" they continue,

\section{Simple images and words capturing profound experiences.}

tions. Practitioners sometimes use graphic medicine as an instrument to deliver information about an illness. Other graphic works are fully developed narratives produced by professional graphic artists and novelists. But there is a lot of grey area in between, populated by physicians and nurses, patients and families, all looking to tell their story.

Green wanted to be an artist as he grew up, but studied medicine instead. Today he teaches a fourth-year elective course called "Graphic Storytelling and Medical Narratives" at Penn State University, one of the first medical schools in North America to include a department of medical humanities. Green notes that "for so many years, medicine has focused on the scientific basis of "readers of comics, like doctors in the exam room, must determine meaning by inferring what happens out of sight and without words." In this way, the doctors who take Green's course can use comics to hone the diagnostic and interpretive skills they need to connect more effectively with patients.

Ian Williams, the Welsh physician who coined the term "graphic medicine" and founded www.graphicmedicine.org, also chose to study medicine over art before finding ways to incorporate graphic narratives into his practice. Williams lauds the interdisciplinary nature of graphic medicine for bringing together "people who would otherwise be working in complete isolation."

He has seen the conference double its enrolment in a mere three years.

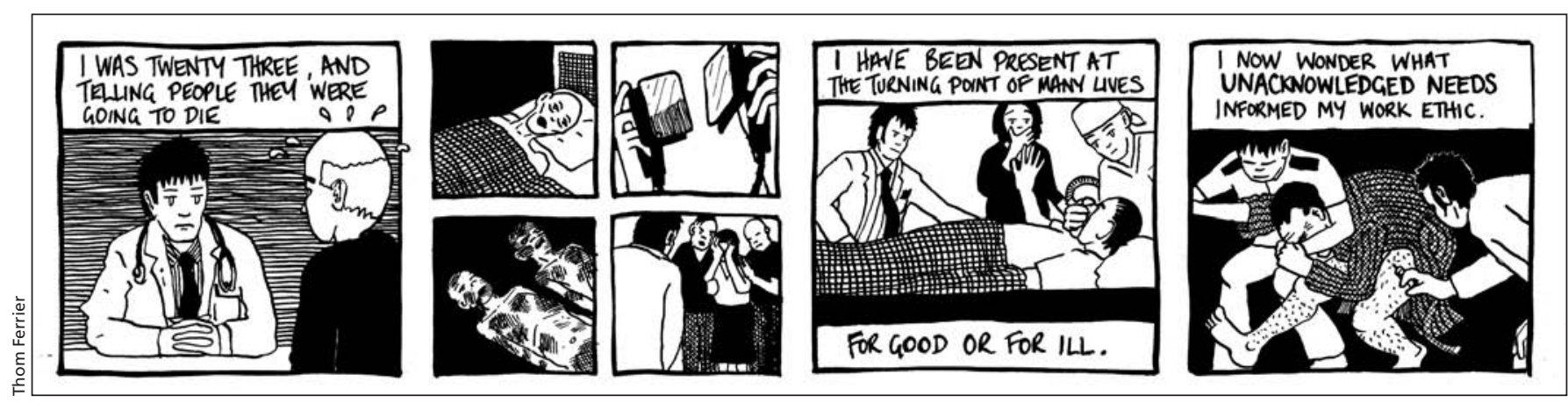

“Tough," by Thom Ferrier (the pen name for lan Williams), 2009 


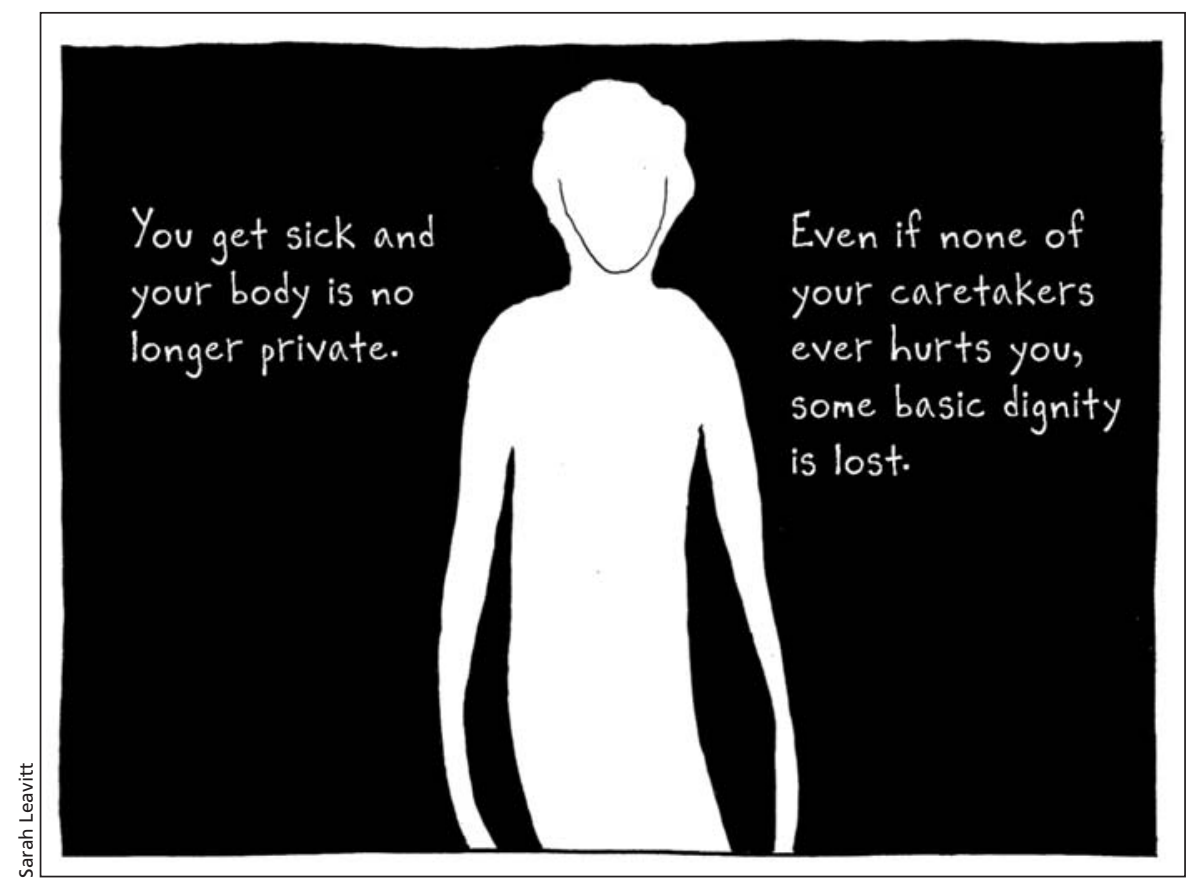

2010 work called "Tangles," by Canadian writer and cartoonist Sarah Leavitt.

"It's providing a liberating platform where traditional barriers are being broken," he says. "Within academia, there's been a tradition of not introducing the 'I', the author, the personal experience. I think we're kind of blowing that away."

The conference, held July 22-24, 2012, attracted about 140 people, encompassing a range of experiences. Graphic medicine pioneer Joyce Farmer who delivered the closing keynote address, gained notoriety for her 1970s underground comic series Tits \& Clits, which addressed sexuality from a female perspective. She and her co-creator Lyn Chevely were providing health counselling for women in 1973, when they decided to create Abortion Eve, a manual to educate women about the procedure of abortion and its associated emotional trauma. The release of the manual, which coincided with the Rowe v. Wade decision, was one of the first known medical comics. At the time, even Planned Parenthood balked at distributing the comic because of its graphic artwork.

For about a decade, MK Czerwiec, who goes by the moniker "Comic

\section{A sampling of graphic medicine links}

- www.graphicmedicine.org Michael Green's students)
Ebenezer Scrooge is supplanted by a callous, jaded physician who has forgotten what it means to care for his patients.

Monk's narrative focuses on the most basic aspects of care: expressing empathy in the face serious illness, calling patients at home, comforting the families who must watch their loved ones suffer. His simple drawings convey a powerful and seemingly obvious message, a message Monk values after watching his partner's grandmother receive unsympathetic care from a doctor as she faced a terminal illness.

Co-organizer and University of Toronto professor Shelley Wall observes that "there's an academic and medical discourse that's about looking at something and describing it. Then there's a creative discourse about experiencing something and giving it voice. Those [discourses] have to meet, and I think that's what's happening at these conferences." Academic and conference organizer Susan Squier agrees, and hopes this kind of creative empathy can inform the medical profession and influence pedagogy more broadly. "There's an interest not just in accepting medicine as it is, but being activist about changing it. That's what excites me about this."

\section{Desmond Cole}

Journalist

Toronto, Ont.

\section{References}

1. Mission statement. New York (NY): Columbia University College of Physicians and Surgeons. Available: www.narrativemedicine.org (accessed 2012 Aug. 27) .

2. Green M, Myers KR. Graphic medicine: use of comics in medical education and patient care. $B M J$ 2010;340:c863.

CMAJ 2012. DOI:10.1503/cmaj.121413

- http://pennstatehershey.org/web/humanities/home/resources/comicbook (work of

- www.theglobeandmail.com/arts/graphic-medicine-in-photos/article4474548/ (The Globe and Mail's feature on women in graphic medicine)

- www.momscancer.com/ (Brian Fies' award-winning memoir) 